\title{
Complicaciones postquirúrgicas y posibles factores de riesgo asociados en pacientes con fracturas diafisarias de fémur o tibia tratados en el Hospital General de Mexicali de enero de 2018 a enero de 2020
}

\author{
Postsurgical complications and possible associated risk factors in patients \\ with diaphyseal fractures of the femur or tibia treated at Hospital \\ General de Mexicali, from January 2018 to January 2020
}

Nadia Estavillo Martínez, * Marlene Vanessa Salcido Reyna, ${ }^{\ddagger}$ Ricardo Iván Quintero Becerra§ *Residente de $4^{\circ}$ año en Traumatología y Ortopedia; $¥$ Médico adscrito del Servicio de Traumatología y Ortopedia; $\$$ Residente de $1^{\circ}$ año en Traumatología y Ortopedia.

Hospital General de Mexicali. México.

\begin{abstract}
Resumen
Globalmente, existe una alta tasa de complicaciones postquirúrgicas en pacientes con fracturas diafisarias de fémur y tibia; sin embargo, éstas varían ampliamente dependiendo de sus factores de riesgo asociados. Con base en la información obtenida en el expediente clínico electrónico se realizó un estudio retrospectivo, con el cual se describieron las principales complicaciones postquirúrgicas en el manejo de fracturas diafisarias de fémur y tibia así como sus factores de riesgo asociados en 144 pacientes atendidos en el Hospital General de Mexicali durante el periodo de enero de 2018 a enero de 2020. Dichas complicaciones fueron la infección (de sitio quirúrgico, material de osteosíntesis u osteomielitis), pseudoartrosis (atrófica o hipertrófica) y fatiga de material de osteosíntesis. Al contar con evidencia sobre la correlación de las complicaciones postquirúrgicas con sus respectivas causas y factores de riesgo asociados, será factible crear nuevos algoritmos de manejo y actualizar las técnicas quirúrgicas empleadas para disminuir significativamente la incidencia de resultados desfavorables a corto, mediano y largo plazo en pacientes del Hospital General de Mexicali.
\end{abstract}

Palabras clave: Fractura, tibia, fémur, infección, pseudoartrosis, fatiga de implante.

\section{Abstract}

Globally, there is a high rate of postsurgical complications in patients with diaphyseal fractures of the femur and tibia, however, these vary widely depending on their associated risk factors. Based on the information obtained in the electronic medical record, a retrospective study was carried out which described the main post-surgical complications in the management of diaphyseal fractures of the femur and tibia, as well as their associated risk factors, in 144 patients treated at Hospital General de Mexicali, during the period from January 2018 to January 2020. Said complications are infection (surgical site, osteosynthesis material or osteomyelitis), pseudoarthrosis (atrophic or hypertrophic) and orthopedic implant failure. By having evidence on the correlation of post-surgical complications with their respective causes and associated risk factors, it will be possible to create new management algorithms and update the surgical techniques used, to significantly reduce the incidence of unfavorable short, medium and long term results in patients of the General Hospital of Mexicali.

Keywords: Fracture, tibia, femur, infection, pseudoarthrosis, implant failure.

Correspondencia:

Dra. Nadia Estavillo Martínez

E-mail: nadia92.em@ hotmail.com

Recibido: 28-12-2021. Aceptado: 01-01-2022.
Citar como: Estavillo MN, Salcido RMV, Quintero BRI. Complicaciones postquirúrgicas y posibles factores de riesgo asociados en pacientes con fracturas diafisarias de fémur o tibia tratados en el Hospital General de Mexicali de enero de 2018 a enero de 2020. Orthotips. 2022; 18 (1): 8-15. https://dx.doi.org/10.35366/103726 


\section{Introducción}

A nivel mundial, existen tres principales complicaciones postquirúrgicas en pacientes que son tratados por fractura diafisaria de fémur o tibia, las cuales son infección, pseudoartrosis y fatiga de material de osteosíntesis. Dichas complicaciones generan un gran costo por el incremento en días de estancia hospitalaria, uso de medicamentos y reintervenciones quirúrgicas.

Las fracturas de la tibia y el peroné son relativamente comunes y se han reconocido como lesiones graves y debilitantes durante siglos. Las descripciones del tratamiento de las fracturas tibiales se incluyen en el Papiro Edwin Smith, un antiguo texto médico egipcio que data de al menos 1,500 a 1,600 años antes de Cristo. Las fracturas tibiales están asociadas con una amplia gama de mecanismos y severidades de lesiones. Aunque la mayoría de las fracturas son cerradas, las fracturas abiertas de la tibia se observan con más frecuencia que en muchos otros huesos debido a su ubicación subcutánea.

El tratamiento de las fracturas de tibia y peroné está influenciado en gran medida por la lesión de tejidos blandos asociada. Las fracturas abiertas graves de la tibia están asociadas con altas tasas de complicaciones y malos resultados a largo plazo. ${ }^{1}$ Aproximadamente más de 2 millones de fracturas ocurren anualmente en los EUA, de ellas 100000 (5\%) desarrollan retardo de consolidación y pseudoartrosis. La incidencia está relacionada con la forma de presentación, la cual varía de manera individual en cada enfermo, los cuales presentan factores de riesgo adicionales que complican el manejo de esta entidad, lo que constituye un verdadero reto para el cirujano ortopédico.

La incidencia y la prevalencia informadas de la falta de unión varían significativamente según la región anatómica y los criterios utilizados para definir la falta de unión. Esta variabilidad refleja la complejidad general de comprender la epidemiología de la pseudoartrosis. Los factores de riesgo de pseudoartrosis se pueden caracterizar como dependientes o independientes del paciente. Los factores de riesgo dependientes del paciente establecidos incluyen edad avanzada, diversas comorbilidades médicas, sexo, tabaquismo, uso de antiinflamatorios no esteroideos, diversos trastornos genéticos, enfermedades metabólicas y deficiencia nutricional. Los factores independientes del paciente incluyen el patrón, la ubicación y el desplazamiento de la fractura, la gravedad de la lesión de los tejidos blandos, el grado de pérdida ósea, la calidad del tratamiento quirúrgico y la presencia o ausencia de infección. ${ }^{2}$

La tibia, especialmente su porción diafisaria, se encuentra expuesta mayormente a esta complicación por varias razones anatómicas como: localización subcutánea, pobre cobertura de músculos y condiciones de vascularidad precaria en su tercio distal. ${ }^{3}$

Antes de la era moderna de los antibióticos y las técnicas quirúrgicas estériles, las fracturas tibiales abiertas ocasionaban amputación o muerte. Se estima que en la guerra de Crimea (1853-1856) la tasa de supervivencia después de las heridas de bala tibiales fue inferior a 20\%. Incluso en la Primera Guerra Mundial, una de cada cinco lesiones de batalla involucraba fracturas inestables de la parte inferior de la pierna, la mayoría de las cuales estaban abiertas. En ese momento, las fracturas tibiales relacionadas con el combate se asociaron con una tasa de mortalidad de aproximadamente $10 \%$ y una tasa de amputación de más de $20 \%$.

Durante muchos años, la tibia fue el hueso largo fracturado con mayor frecuencia y en muchos países éste sigue siendo el caso. Sin embargo, no es una fractura por fragilidad y en los países desarrollados el aumento en las fracturas por fragilidad y fracturas periprotésicas ha significado que la incidencia de fracturas diafisarias femorales ha aumentado, por lo que la incidencia de fracturas diafisarias femorales y tibiales actualmente es muy similar. En comparación con las fracturas en otras partes del cuerpo, las fracturas tibiales tienen tasas relativamente altas de no unión y mala unión. La diáfisis tibial es el sitio más común de fractura en la tibia y alrededor de $80 \%$ de estas lesiones tienen fracturas peroneas asociadas. Los datos publicados sugieren una incidencia de 17 por 100,000 personas al año, aunque los datos más recientes indican que la incidencia puede estar disminuyendo.

Las complicaciones a largo plazo más comunes en las fracturas expuestas diafisarias de la tibia son infección y no unión. Se ha informado que las tasas de infección de fracturas tibiales abiertas están entre 21.1 y $45.7 \%$, dependiendo de la gravedad de la lesión de acuerdo con el sistema de clasificación GustiloAnderson. Con el uso de antibióticos tempranos las tasas de desbridamiento están disminuyendo. ${ }^{4}$

Un desbridamiento completo, lavado y estabilización esquelética son los pilares del tratamiento inicial junto con antibióticos intravenosos. Estudios recientes han defendido el papel de la cobertura temprana de la herida como un medio para disminuir la infección de la herida. ${ }^{5}$ 
La incidencia de infección por fracturas en la diáfisis de la tibia en países desarrollados, son del $1.9 \%$ en fracturas cerradas y del $8.8 \%$ en fracturas abiertas. ${ }^{6}$

Estas fracturas también tienen una alta incidencia de no unión debido a la pérdida del suministro de sangre, la presencia de infección o la fijación inadecuada.

El tiempo de unión por lo general toma de tres a seis meses, después de lo cual el tratamiento dependerá de la causa de la no unión. Las no uniones sépticas necesitarán un tratamiento más agresivo de la infección, la no unión hipertrófica requerirá mejor estabilización y la no unión atrófica necesitará injerto óseo o escariado adicional para estimular la formación de hueso. ${ }^{7}$

Biomecánica es la aplicación de las leyes de la mecánica en los seres vivos; por lo tanto, la biomecánica abarca todas las acciones que se ejercen sobre la estructura de soporte del cuerpo, las cargas, los esfuerzos y la respuesta a los mismos, así como las deformaciones plástica y elástica, con sus consecuencias y efectos a corto, mediano y largo plazo. De igual manera, estudia el movimiento del cuerpo en el espacio, la marcha, carrera, salto, entre otros. También contempla el comportamiento de los implantes, el organismo en el cual se aplican, los mecanismos de lesión con sus características y la cinética del traumatismo.

Definición de principio biomecánico en osteosíntesis: es la forma en la cual funcionan o interactúan el o los implantes y el hueso en el cual son aplicados para el tratamiento quirúrgico de las fracturas. ${ }^{8}$

La osteosíntesis puede definirse como la estabilización temporal interna o externa de los fragmentos óseos, hasta que se obtiene la consolidación. Los materiales utilizados para los implantes de osteosíntesis deben ser biocompatibles y proporcionar elevadas prestaciones mecánicas. ${ }^{9}$

Las placas ortopédicas metálicas se utilizan para fijar fragmentos óseos fracturados u osteotomizados. Al puentear una fractura u osteotomía, existe el riesgo de falla de la osteosíntesis si el hueso no se une en el tiempo suficiente como para que la fatiga provoque la pérdida de la fijación a través de la rotura de la placa o el tornillo, o de lo contrario, la extracción de la construcción. Se ha demostrado que la configuración del tornillo, la longitud del tornillo (monocortical frente a bicortical), el material de la placa y la calidad de fabricación de la placa tienen efectos sobre la resistencia, la rigidez y la resistencia a la fatiga de la estructura de la placa. ${ }^{10}$
La fijación interna de las fracturas diafisarias de tibia mediante fijación con placa no suele considerarse el tratamiento de elección debido a las complicaciones que eventualmente pueden surgir; sin embargo, las placas de osteosíntesis son una alternativa viable al enclavado intramedular y sus resultados pueden ser favorables si se aplican correctamente los principios de estabilidad, el manejo de los tejidos blandos y las técnicas quirúrgicas adecuadas. ${ }^{11}$

El enclavado intramedular se ha convertido en un procedimiento estándar para el tratamiento de fracturas cerradas de huesos largos, ofreciendo un excelente potencial de cicatrización y una baja tasa de complicaciones. ${ }^{12}$

Las fracturas de la diáfisis femoral se observan en todos los grupos de edad y son atribuibles a una variedad de mecanismos. Suele haber una distribución bimodal de las fracturas relacionada con la edad y el sexo, y las lesiones ocurren con mayor frecuencia en hombres jóvenes después de un traumatismo de alta energía y en mujeres ancianas por caídas. Los mecanismos en pacientes jóvenes tienden a ser choques de vehículos motorizados, choques de motocicletas, peatones atropellados por vehículos o caídas de altura. La distribución relativa de estas fracturas depende de múltiples factores, incluida la ubicación geográfica y el país de estudio. La incidencia de fracturas de la diáfisis femoral fue de 9.9 por 100,000 personas/año.

La evaluación y el tratamiento de los pacientes con fracturas de la diáfisis femoral continúan evolucionando sobre la base de una mejor comprensión de la anatomía local, el impacto del tratamiento y la biomecánica de las técnicas de fijación. A partir de la introducción del clavo intramedular por Kuntscher durante los años posteriores a la Segunda Guerra Mundial, la supervivencia del paciente y los resultados han seguido mejorando. La prevención y el tratamiento mejorados del acortamiento, la angulación, la infección y la pseudoartrosis de las fracturas han hecho del clavo intramedular el tratamiento principal para la mayoría de las fracturas de la diáfisis femoral.

El enclavado intramedular ha sido durante mucho tiempo el estándar de oro en el tratamiento de las fracturas femorales diafisarias. Este tratamiento en las fracturas femorales abiertas da como resultado una unión satisfactoria y tasas de infección aceptables comparables a las observadas en el tratamiento de las fracturas femorales cerradas. Las lesiones de 
grado III de Gustilo demuestran una tasa de infección profunda más alta y se recomienda el cumplimiento estricto de los protocolos quirúrgicos establecidos de desbridamiento y fijación. ${ }^{13}$

La infección del sitio quirúrgico relacionada con el enclavado intramedular se considera una complicación grave y de difícil tratamiento, que puede provocar un retraso en la consolidación y pérdida de la función de la extremidad o incluso la amputación. La incidencia reportada de esta complicación oscila entre $0.9 \%$ y $23 \%$ y es mayor en estudios que incluyen pacientes con fracturas expuestas. ${ }^{14}$

Las infecciones quirúrgicas aparte de dividirse en infecciones superficiales, profundas y de órgano/ espacio, se dividen en dos principales grupos: infección del sitio quirúrgico e infecciones que requieren intervención quirúrgica, además del tratamiento antimicrobiano para resolverse. La infección asociada

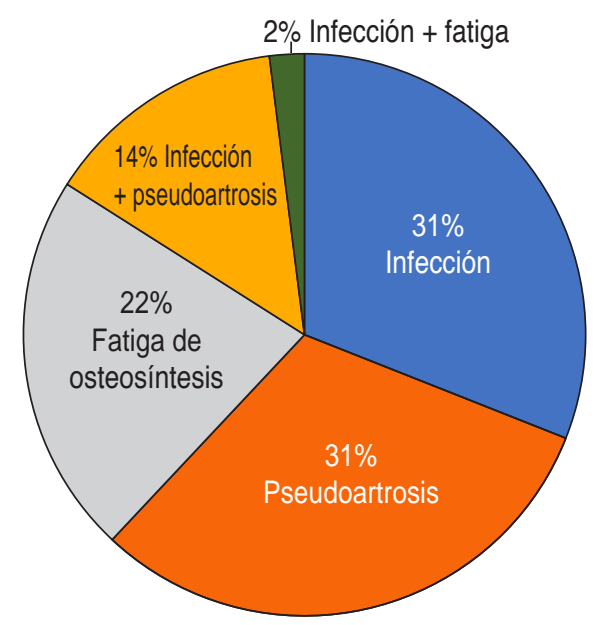

Figura 1: Pacientes complicados $(\mathrm{N}=42)$. a dispositivos ortopédicos (IADO) corresponde al grupo que requiere intervención quirúrgica para su resolución y se describe como: 1) la presencia de trayectos fistulosos que comunican el espacio periférico hacia al dispositivo; o 2) un cultivo positivo por lo menos en dos muestras quirúrgicas de material tomado de tejidos transquirúrgicos, o 3) aspirado articular; o 4) la presencia de neutrófilos en especímenes de tejido; o 5) la presencia de material purulento durante la cirugía. ${ }^{15}$

A nivel mundial, existen tres principales complicaciones postquirúrgicas en pacientes que son tratados por fractura diafisaria de fémur o tibia, las cuales son infección, pseudoartrosis y fatiga de material de osteosíntesis. Dichas complicaciones generan un gran costo por el incremento en días de estancia hospitalaria, uso de medicamentos y reintervenciones quirúrgicas.

Durante el periodo de enero de 2018 a enero de 2020, en el Hospital General de Mexicali se dio manejo quirúrgico a 144 pacientes con los diagnósticos de fractura diafisaria de fémur y de tibia, de los cuales existe una cifra importante de las complicaciones antes mencionadas, por lo que se realizó un estudio transversal analítico con el objetivo de investigar la incidencia de complicaciones y sus factores de riesgo asociados, y así crear un desenlace más favorable para las futuras atenciones.

\section{Material y métodos}

Diseño de estudio: transversal, analítico.

\section{Criterios de inclusión:}

1. Pacientes con fractura diafisaria de tibia.

2. Pacientes con fractura diafisaria de fémur.

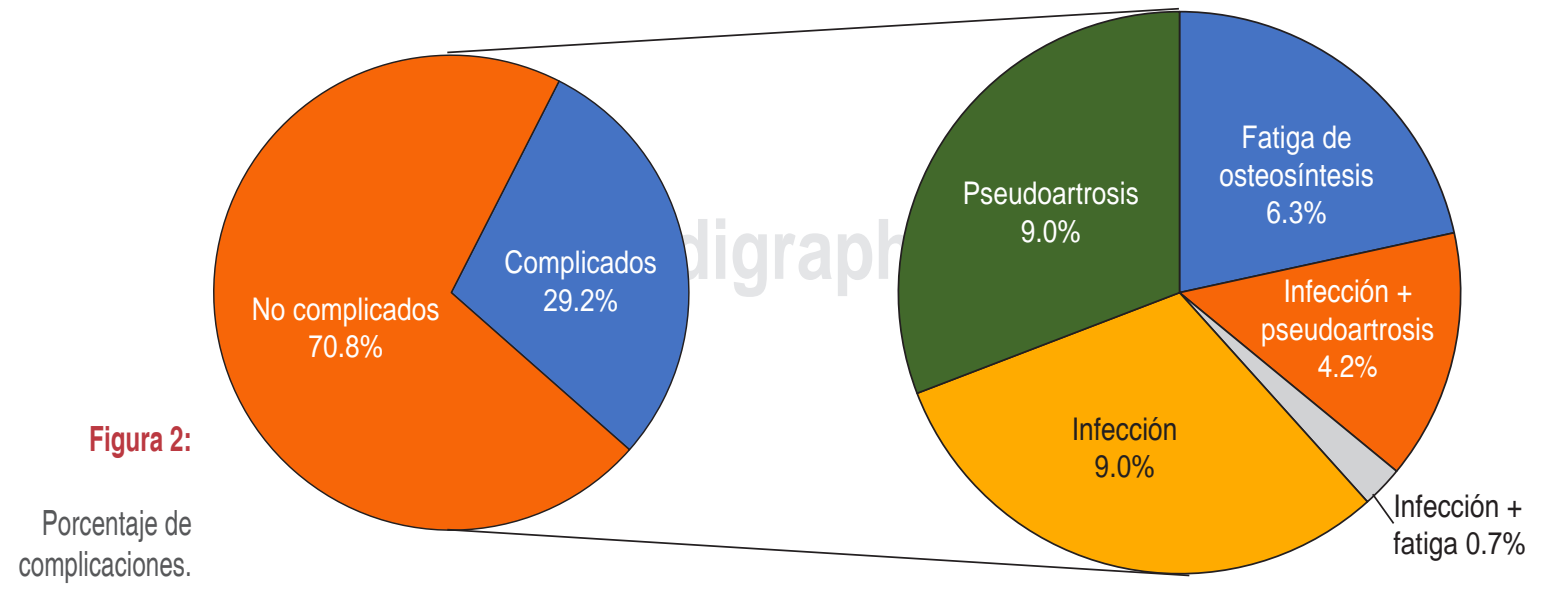




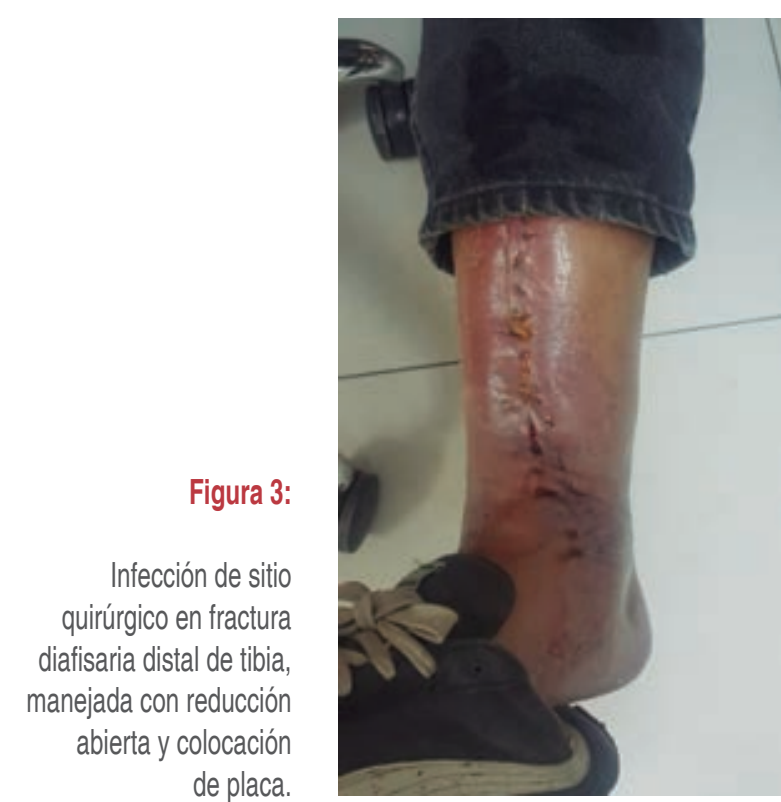

3. Pacientes atendidos en Hospital General de Mexicali.

4. Pacientes manejados con tratamiento quirúrgico.

\section{Criterios de exclusión:}

1. Pacientes referidos o tratados inicialmente en otra institución.

2. Pacientes con fracturas articulares de tibia o fémur.

\section{Criterios de eliminación:}

1. Pacientes tratados de manera conservadora.

2. Pacientes no tratados, ya sea por alta voluntaria, defunción, fuga o traslado a otra institución.

3. Pacientes sin seguimiento en consulta externa.

a. Universo de estudio: pacientes sin distinción de sexo y edad que fueron atendidos en el Hospital General de Mexicali por diagnósticos de fractura diafisaria de fémur o tibia en el periodo de enero de 2018 a enero de 2020 y se manejaron con tratamiento quirúrgico. Se obtuvo una muestra de 144 pacientes.

b. Técnicas quirúrgicas: reducción cerrada versus abierta y fijación interna con clavo centromedular para tibia, reducción abierta y fijación interna con placa para tibia, reducción cerrada y colocación de fijador externo para tibia, reducción cerrada versus abierta y fijación interna con clavo centromedular anterógrado para fémur, reducción cerrada versus abierta y fijación interna con clavo centromedular retrógrado para fémur, reducción abierta y fijación interna con placa para fémur, reducción cerrada y colocación de fijador externo para fémur.

\section{Resultados}

Se obtuvo una muestra de 144 pacientes, de los cuales 42 presentaron complicaciones (29.2\%) y 102 sin complicaciones $(70.8 \%)$ (Figuras 1 y 2 ).

118 hombres y 26 mujeres. Edad promedio de 33.2 años, 85 fracturas de tibia y 59 de fémur, 48 fracturas expuestas y 96 no expuestas, 14.2 días de estancia hospitalaria promedio, 99 procedimientos quirúrgicos abiertos y 45 a foco cerrado.

20 pacientes desarrollaron un proceso infeccioso (13.8\%), de los cuales 13 tuvieron sólo infección, seis se acompañaron de pseudoartrosis y uno de fatiga de material de osteosíntesis (Figura 3).

De estos 20 pacientes, se encontró que: 19 fueron del sexo masculino, 13 fracturas de tibia y siete de fémur, 12 fracturas expuestas y ocho no expuestas, 14 días de estancia hospitalaria promedio, 10 con tabaquismo positivo, 13 con etilismo positivo, ocho usuarios de drogas, seis causados por caída de motocicleta, 14 fueron sometidos a cirugía abierta y seis a foco cerrado.

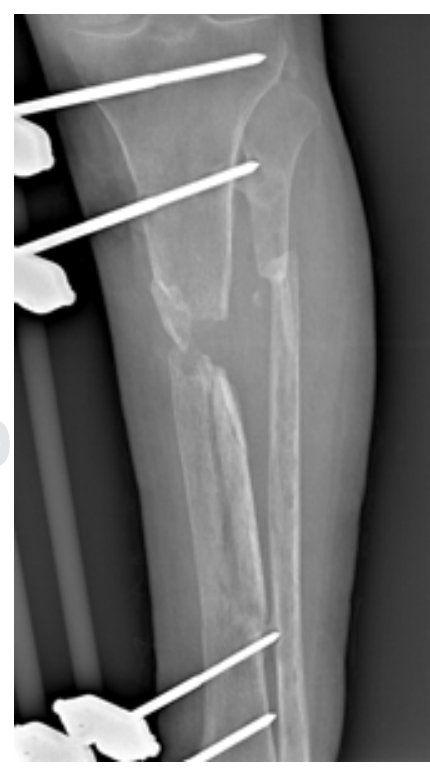

Figura 4:

Radiografía anteroposterior de tibia izquierda en la cual se observa pseudoartrosis atrófica secundaria a una fractura expuesta diafisaria. 


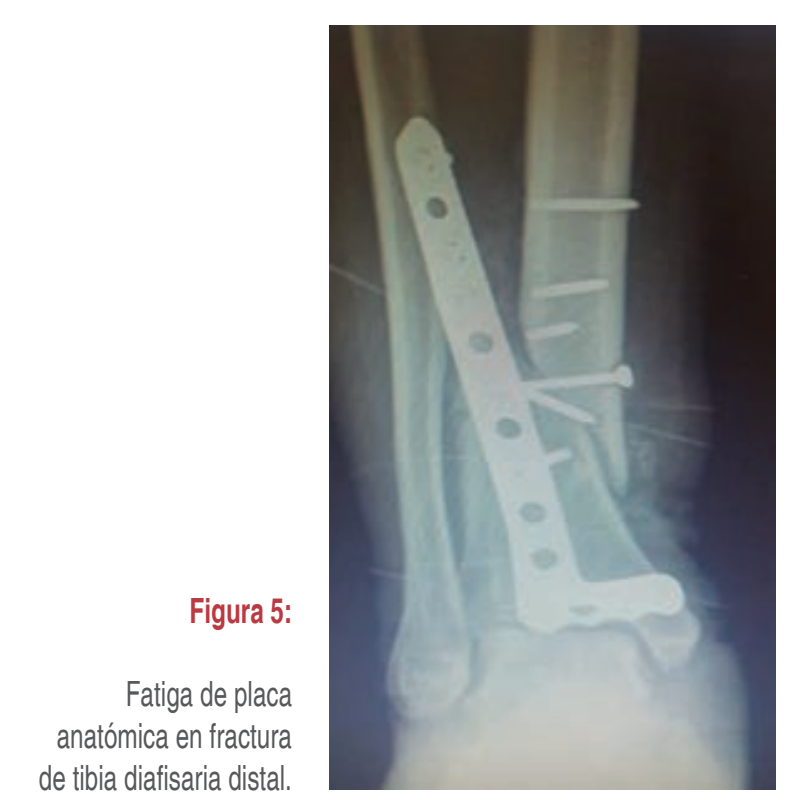

19 pacientes desarrollaron pseudoartrosis (13.2\%), de los cuales 13 presentaron sólo pseudoartrosis y seis se acompañaron de infección (Figura 4).

De ellos, 15 fueron del sexo masculino, 11 fracturas de tibia y ocho de fémur, nueve fracturas expuestas y 10 no expuestas, 13.8 días de estancia hospitalaria promedio, siete con tabaquismo positivo, 11 con etilismo positivo, cuatro usuarios de drogas, ocho causados por choque automovilístico y siete por caída de motocicleta, ocho sometidos a cirugía abierta y 11 a foco cerrado.

10 pacientes mostraron fatiga de material de osteosíntesis $(6.9 \%)$, de los cuales uno se acompañó de infección (Figura 5).

De ellos, nueve fueron del sexo masculino, seis fracturas de tibia y cuatro de fémur, una fractura expuesta y nueve no expuestas, 13.4 días de estancia hospitalaria promedio, siete con tabaquismo positivo, cinco con etilismo positivo, ocho usuarios de drogas, cuatro fueron fracturados por atropellamiento, los 10 pacientes fueron sometidos a cirugía abierta. En siete de ellos se utilizó como implante DCP (placas de compresión dinámica) de $4.5 \mathrm{~mm}$, en dos se utilizó placa anatómica para tibia distal y en uno, placa convencional tipo palo de hockey en fémur.

Fractura expuesta/no expuesta:

$\mathrm{OR}=2.17, \mathrm{IC}=1.02-4.6, \mathrm{p}=0.04$.

Cirugía abierta/cerrada:

$\mathrm{OR}=0.94, \mathrm{IC}=0.43-2.05, \mathrm{p}=0.02$.

\section{Discusión}

De acuerdo con los objetivos de nuestro estudio, se demostró una incidencia de $29.2 \%$ de complicaciones postquirúrgicas en pacientes con fractura diafisaria de fémur o tibia tratados en el Hospital General de Mexicali durante el periodo de enero de 2018 a enero de 2020. Comparando estos resultados con los observados en revisiones sistemáticas a nivel mundial, existe una diferencia estadísticamente significativa en la incidencia de complicaciones, siendo mayor en nuestra población, la cual presenta una incidencia de infección de $13.8 \%$ frente a 6-12\% en estudios globales; y pseudoartrosis en $13.2 \%$ versus 3-8.4\%., ${ }^{5,6}$ Aún no existe un consenso sobre la incidencia de fatiga de material de osteosíntesis en fracturas diafisarias de fémur o tibia y sus factores de riesgo asociados. En nuestro estudio se reportó en $6.9 \%$.

Los principales factores de riesgo asociados con infección fueron: sexo masculino (95\%), cirugía abierta (70\%), fractura de tibia (65\%), etilismo (65\%), fractura expuesta $(60 \%)$, tabaquismo $(50 \%)$, uso de drogas (40\%), mecanismo de lesión por caída de motocicleta (30\%).

Los principales factores de riesgo asociados con pseudoartrosis fueron: sexo masculino (79\%), mecanismo de lesión por choque automovilístico o caída de motocicleta (79\%), fractura de tibia (57\%), etilismo (57\%), fractura expuesta (47\%), cirugía abierta (42\%), tabaquismo (36\%).

Los principales factores de riesgo asociados con fatiga de material de osteosíntesis fueron: cirugía abierta con colocación de placa (100\%), sexo masculino (90\%), uso de drogas (80\%), tabaquismo (70\%), fractura de tibia (60\%).

No se observó ningún caso de fatiga de material de osteosíntesis al utilizar clavo centromedular para fémur o tibia, en cambio, en la totalidad de casos de falla de implante se utilizó placa.

De 99 pacientes sometidos a cirugía abierta, se complicaron 28 (28.2\%). De 45 pacientes sometidos a cirugía a foco cerrado, se complicaron 14 (31.1\%), por lo que la técnica quirúrgica abierta se considera un factor de riesgo principalmente asociado con infección más que con pseudoartrosis.

De 48 pacientes con fractura expuesta, 19 presentaron complicaciones (39.5\%). De 96 fracturas no expuestas, se complicaron 23 (23.9\%).

La fractura expuesta se considera un factor de riesgo asociado con complicaciones, principal- 
mente con infección, siendo proporcional a su grado de severidad.

No hubo diferencia significativa en los días de estancia hospitalaria entre los pacientes complicados y los no complicados.

Se observó que las toxicomanías son un factor de riesgo asociado en todas las complicaciones.

No se encontró una asociación significativa de las complicaciones postquirúrgicas con las comorbilidades de diabetes mellitus y trastornos psiquiátricos, por lo que no se consideran factores de riesgo principales relacionados.

Los mecanismos de lesión que se consideraron en el estudio fueron: baja energía, choque automovilístico, caída de motocicleta, atropellamiento, herida por proyectil de arma de fuego, otros.

Los tres principales mecanismos asociados con complicaciones fueron: caída de motocicleta, choque automovilístico y atropellamiento.

En cuanto al segmento óseo, las fracturas diafisarias de tibia presentaron complicaciones en 30.5\% de los casos y las fracturas diafisarias de fémur en $27.1 \%$. Sin embargo, fue más frecuente la fractura de tibia $(59 \%)$ que la de fémur (41\%).

\section{Conclusiones}

Las complicaciones postquirúrgicas en pacientes con fractura diafisaria de fémur o tibia en el Hospital General de Mexicali han sido una problemática que se ha hecho más notoria en los últimos años. Por ello, se realizó un estudio transversal en el que se logró demostrar una incidencia de $29.2 \%$ de complicaciones, lo cual supera las cifras promedio reportadas en diversos estudios globales.

Se observó una incidencia de infección en 13.8\%, con sus principales factores de riesgo asociados: sexo masculino, cirugía abierta, fractura de tibia, etilismo, fractura expuesta, tabaquismo, uso de drogas, mecanismo de lesión por caída de motocicleta.

La pseudoartrosis se presentó en $13.2 \%$ de los pacientes, teniendo como principales factores de riesgo asociados: sexo masculino, mecanismo de lesión por choque automovilístico o caída de motocicleta, fractura de tibia, etilismo, fractura expuesta, cirugía abierta, tabaquismo.

La fatiga de material de osteosíntesis ocurrió en $6.9 \%$ de los casos. Sus principales factores de riesgo asociados fueron: cirugía abierta con colocación de placa, sexo masculino, uso de drogas, tabaquismo, fractura de tibia.
En cuanto al tratamiento de este tipo de fracturas, podemos concluir que se recomienda realizar reducción a foco cerrado, cuando sea posible, principalmente para disminuir la incidencia de infección así como de pseudoartrosis al evitar la desperiostización y devascularización y realizar la fijación con clavo centromedular para reducir el riesgo de fatiga de material de osteosíntesis observado con el uso de placas.

Coincidiendo con múltiples revisiones sistemáticas, las fracturas expuestas demostraron ser un factor de riesgo asociado con complicaciones, por lo que se consideran una urgencia quirúrgica. Se debe iniciar profilaxis antibiótica así como realizar un aseo quirúrgico y desbridamiento lo antes posible para disminuir el riesgo de complicaciones.

Dado que se observó una incidencia significativa de complicaciones en pacientes de sexo masculino con toxicomanías positivas, así como los que sufrieron mecanismos de lesión de alta energía, se recomienda considerar estos factores como mayor riesgo de una evolución desfavorable, y tomar decisiones terapéuticas con más cautela para prever posibles complicaciones.

\section{Referencias}

1. Hungria JOS, Mercadante MT. Fratura exposta da diáfise da tíbia - tratamento com osteossíntese intramedular após estabilização provisória com fixador externo nao transfixante. Ver Bras Ortop. 2013; 48 (6): 482-490.

2. Hak DJ, Fitzpatrick D, Bishop JA, Marsh JL, Tilp S, Schnettler $\mathrm{R}$, et al. Delayed union and nonunions: epidemiology, clinical issues, and financial aspects. Injury. 2014; 45 Suppl 2: S3-S7.

3. Gutiérrez Blanco MO, Leyva Basterrechea F, Álvarez López A. Retardo de consolidación y seudoartrosis de la tibia. Rev Cub Med Mil. 2009; 38 (1).

4. Court-Brown CM, Keating JF, McQueen MM. Infection after intramedullary nailing of the tibia. Incidence and protocol for management. J Bone Joint Surg Br. 1992; 74 (5): 770-774.

5. Singh J, Rambani R, Hashim Z, Raman R, Sharma HK. The relationship between time to surgical debridement and incidence of infection in grade III open fractures. Strategies Trauma Limb Reconstr. 2012; 7 (1): 33-37.

6. Doshi P, Gopalan H, Sprague S, Pradhan C, Kulkarni S, Bhandari $\mathrm{M}$. Incidence of infection following internal fixation of open and closed tibia fractures in India (INFINITI): a multicentre observational cohort study. BMC Musculoskelet Disord. 2017; 18 (1): 156.

7. Pretell Mazzini JA, Ruiz Semba C, Rodríguez Martín J. Trastornos de la consolidación: retardo y pseudoartrosis. Rev Med Hered. 2009; 20 (1): 31-39.

8. Ramos-Maza E, García-Estrada F, Domínguez-Barrios C, Chávez-Covarrubias G, Meza-Reyes G, Buffo-Sequeira I. Principios biomecánicos para la osteosíntesis, re-evolución. Acta Ortop Mex. 2016; 30 (Suppl: 1): 1-8.

9. Altmann M, Cognet JM, Eschbach L, Gasser B, Richards G, Simon P. Materiales utilizados en la osteosíntesis. EMC - 
Técnicas Quirúrgicas - Ortopedia y Traumatología. 2009; 1 (1): 1-8.

10. Parr WCH, Wang T, Tan C, Dan MJ, Walsh WR, Morberg P. Fatigue implications for bending orthopaedic plates. Injury. 2021; 52 (10): 2896-2902.

11. Concha Sandoval JM, Osma Rueda JL, Sandoval Daza A. Management of diaphyseal tibial fractures by plate fixation with absolute or relative stability: a retrospective study of 45 patients. Trauma Surg Acute Care Open. 2017; 2 (1): e000029.

12. Malik MH, Harwood P, Diggle P, Khan SA. Factors affecting rates of infection and nonunion in intramedullary nailing. J Bone Joint Surg Br. 2004; 86 (4): 556-560.

13. Saleeb H, Tosounidis T, Papakostidis C, Giannoudis PV. Incidence of deep infection, union and malunion for open diaphyseal femoral shaft fractures treated with IM nailing: a systematic review. Surgeon. 2019; 17 (5): 257-269.

14. Oliveira PR, Leonhardt MC, Carvalho VC, Kojima KE, Silva JS, Rossi F, et al. Incidence and risk factors associated with infection after intramedullary nailing of femoral and tibial diaphyseal fractures: prospective study. Injury. 2018; 49 (10): 1905-1911.

15. Guía de Práctica Clínica. Diagnóstico y tratamiento de las Infecciones Asociadas a Dispositivos das a Dispositivos Ortopédicos Prótesis y/o material de osteosíntesis. México: Instituto Mexicano del Seguro Social; 2013.

\section{Conflicto de intereses}

Sin conflicto de intereses. 